Capítulo 1

\title{
DEMOGRAFÍA DEL ENVEJECIMIENTO Y VEJEZ EN EL MARCO DE LA POLÍTICA PÚBLICA 2007-2019
}

Guadalupe Margarita Cardeño Sanmiguel

Yolanda Rosa Morales Castro

\section{EL SENTIDO DE VIVIR HASTA LA VEJEZ}

El sentido de vivir hasta la vejez ha permitido tener unos lentes finos con vidrios claros para ver la realidad de las personas mayores en Colombia. Este grupo etario lleno de un cúmulo de experiencia, sentimientos, con arraigo familiar y muchos con deseos de continuar luchando por su supervivencia, nos dicen, 'estamos aquí no como sujetos pasivos sino como sujetos activos que podemos dar y recibir de nuestras familias, de nuestro territorio, de nuestro país, con corresponsabilidad social'. Ellos aclaman vivir en democracia, no quieren que sus opiniones se cercenen, quieren participar y ser tenidos en cuenta, que se les consulte sobre su realidad porque son sujetos políticos y quieren opinar sobre las decisiones que se tomen respecto a ellos ya que son una fuerza viva e interesante.

La vejez es una etapa en la cual la persona advierte una diversidad de cambios, que si bien dependen del estilo y calidad de vida que ha llevado a lo largo del transcurso del ciclo, así como del sistema social y familiar en que se han desenvuelto, influyen en los diferentes ámbitos de su vida. De acuerdo con el estereotipo cultural, la vejez es la perdida de capacidades, especialmente las relacionadas con el 
desarrollo físico, lo que en un medio en que la belleza se venera, hace que los individuos experimenten temor y miedo por esa etapa de la vida, lo que abona el camino al aislamiento social; de ahí la importancia de la existencia de sistemas de redes sociales, familiares y de salud que faciliten la integración social de los mayores y garanticen su ciudadanía activa.

Los cambios físicos, producto de la disminución progresiva del funcionamiento biológico, llevan a que la persona mayor pierda paulatinamente autonomía y se amplíe su necesidad de dependencia, con las consecuencias que esto implica en el entorno familiar y social. La funcionalidad en los mayores se evidencia en la capacidad de realizar las actividades de vida diaria y pueden atender y enfrentar las demandas del entono, viviendo independientemente. La disminución o pérdida de las habilidades funcionales afecta de manera significativa los roles y tareas socialmente definidas para las personas; en este momento la socialización del adulto mayor tiende a disminuir, lo que da paso a la discapacidad.

Las actividades diarias como vestirse, bañarse, utilizar los servicios, o en la disminución de la autonomía para consumir alimentos al ser realizadas con la asistencia de un tercero, hace que se aumente la 14 dependencia y a la vez ocasiona sentimientos de frustración, inutilidad y tristeza por la pérdida de control sobre la vida propia. Si a lo anterior se aúna la disminución de la participación en los sistemas sociales, la marginación social se refuerza y se pierde el interés y la confianza de que el sistema social pueda generar alguna satisfacción. De esta manera, el proceso de socialización se ve afectado en esta etapa por la disminución inicial del contacto con familiares y amistades, relacionada con las limitaciones que aparecen a nivel de salud y que pueden perjudicar el contacto con otros. 
En este sentido, la interacción social se convierte en un factor de protección para la salud, bajo la consideración de que ello entraña la existencia de vínculos estrechos con otros y cierto tipo de actividad constante. Esta situación genera a su vez vínculos con otros grupos sociales, lo que permite el mejoramiento y/o sostenimiento de la calidad de vida de los adultos mayores. Es así como el contacto permanente con otros, producto de una socialización activa, permite desarrollar potencialidades que contribuyen al bienestar de los individuos. Señalan Durán et al, que:

(...)la integración social depende de las redes de apoyo con las que cuente el adulto mayor, entendiendo éstas como aquellas relaciones significativas que una persona establece cotidianamente y a lo largo de su vida, específicamente con familiares más cercanos, amigos, compañeros de trabajo, pareja, entre otros. Se ha observado que tales redes juegan un papel vital en la adultez mayor, convirtiéndose en el sustento emocional que permite en muchos casos sobrepasar las crisis que se puedan presentar y además, que los adultos mayores sean reconocidos como personas, mejorando su autoestima, su autopercepción, la eficacia personal y, por ende, la identidad. (1)

Cobra significativa importancia la relación existente entre salud, habilidades funcionales y la integración social, lo que hace suponer el diseño e implementación de estrategias de intervención que articulen diversas actividades sociales, que les permitan a los adultos mayores mejorar sus condiciones físicas y su autonomía personal, así como la posición y satisfacción frente a la vida.

Sostiene la Organización Mundial de la Salud OMS (2) que las habilidades funcionales de los mayores son el resultado de la disminución de los diferentes sistemas biológicos; sin embargo, no se puede dejar de lado la interacción de esta variable con la escolaridad, el estilo de vida, el sistema familiar, económico y social, así como con 
variables subjetivas como la percepción de calidad de vida que tenga la persona.

Es importante señalar que en esta etapa de la vida la integración social logra compensar la pérdidas inherentes a la edad; se considera que la interacción y la integración social son factores de protección frente a la aparición de patologías, logrando que aumente su capacidad cognitiva y tenga mejores habilidades de afrontamiento, module su afecto, aumente su calidad de vida y, por ende, su autonomía. Lo anterior tiene como consecuencia el aumento del nivel de movilidad, de independencia, y el mejoramiento del autoconcepto. Sin embargo, los beneficios que puede producir una adecuada integración social están mediados por la pérdida de la salud, la no adaptación de esta etapa, la idea de dependencia física y económica de los hijos, y el no poder realizar las mismas actividades de antes, es así como las habilidades funcionales están asociadas con el nivel de integración social que tenga el adulto mayor, pues a medida que aumenta su edad se amplían las posibilidades de pérdidas, y paralelo a ello, se incrementa la posibilidad de perder las redes sociales de apoyo emocional e instrumental.

16 En Latinoamérica y el Caribe se han presentado importantes cambios demográfico en el siglo XX, viéndose un crecimiento de los grupos de personas mayores con 60 años de edad y más, los cuales se caracterizan por una gran variabilidad en las condiciones socioeconómicas. Es por esto que, desde la década de 1970, las Naciones Unidas se han preocupado por conocer los contextos del envejecimiento de la población mundial y promover acciones entre sus agencias, las instituciones gubernamentales y no gubernamentales de los países que representan (3). 
Colombia, al igual que el resto de países latinoamericanos, ha experimentado desde el siglo XX una rápida transición demográfica. Esto ha afectado la estructura por edad de su población y ha iniciado un proceso de envejecimiento, encontrándose el país en una etapa de envejecimiento moderado. Entre 1985 y 2014 la población colombiana aumentó aproximadamente un $52 \%$, de 31 millones de personas pasó a 48 millones. Se estima que en las próximas tres décadas seguirá creciendo, de forma menor, pero en el 2050 habrá 61 millones de personas (4).

Las causas del envejecimiento en Colombia son el aumento de la esperanza de vida, disminución de la mortalidad, la que la ubica en un territorio envejeciente con una expectativa de vida al nacer de 76 años, superior a la de América Latina que es de 74,7, y países en desarrollo 80 años (5).

La población mayor de Colombia es de 5.750 .000 personas. La más reciente encuesta de Salud, Bienestar y Envejecimiento (SABE) del año 2015, hecha por el Ministerio de Salud y Protección Social, indica que 700.000 de ellos son víctimas de violencia sicológica, 529.000 de negligencia, 195.000 sufren violencia física, 86.000 violencia financiera (explotación económica o control de recursos) y 19.578 violencia sexual (5).

La Encuesta de Demografía y Salud (ENDS) (2015) de Pro familia indica que el objetivo de este estudio es sugerir un conjunto de indicadores de calidad de vida que considere diferencias generacionales, regionales y de género; demás concluyó que Colombia vive actualmente un proceso de transición demográfica plena, en el cual han influido el descenso en la mortalidad, la paulatina disminución de la fecundidad y también las migraciones. Dicho proceso es geográficamente diferencial (6). 
Las situaciones más problemáticas del adulto mayor en Colombia, son el índice de analfabetismo más altos (20 \%) y menores niveles de educación formal; la inseguridad económica de más del 70 \% de la población mayor de 60 años los ha llevado a buena parte a trabajar por necesidad y en general en condiciones de informalidad, de incertidumbre e inequidad en la remuneración, las cuales se alejan de los criterios de trabajo decente planteados por la OIT.

Dicha inseguridad económica tiene claros patrones de inequidad social por estatus, género y territorio.

La encuesta ENDS, 2015, refiere 3 prioridades de Orientación tales como participación en, salud, bienestar, y entornos físicos propicios para el desarrollo pleno del adulto mayor. El estudio tomó como muestra personas mayores de 60 años que respondieron el cuestionario de "Adulto Mayor"; participaron 17.574 personas (6).

Los resultados obtenidos revelan que el 50 \% de la población vieja percibe su salud como buena, y en general no refiere ningún tipo de limitación para realizar actividades de la vida cotidiana. Los peores resultados con relación a percepción de salud y limitación en actividades de la vida cotidiana se encuentran en quienes trabajan en el hogar, sin remuneración y por cuenta propia. Además, se evidencian barreras para la garantía del derecho a la salud: accesibilidad, disponibilidad, calidad.

En cuanto al factor de relación familiar la encuesta arrojó que el $60 \%$ de los hogares cuenta con personas mayores de 60 años, una de ellas era jefe de hogar, y más del 40 \% sostenía económicamente a 2 o 3 personas; y en las viviendas y entornos físicos hay un gran descuido en lo que se refiere al vivir-envejecer, y sobre todo a la vejez y a las personas viejas (6) . 
Además otros estudios realizados por la Universidad de La Sabana, en Bogotá, indican que el 26 \% de las personas mayores de 65 años en el país está pensionada. La depresión que padece el $40 \%$ de esta población, es la segunda patología más frecuente en los adultos mayores después de la hipertensión arterial (7).

De acuerdo con el estudio, los altos índices de la enfermedad se deben a la inequidad económica, ya que los adultos mayores no tienen una pensión social universal no contributiva, muchos viven en pobreza extrema, son víctimas de violencia, maltrato, abuso y tienen un acceso al sistema de salud muy deficiente.

Por otro lado, según el diagnóstico de los adultos mayores en Colombia de la Fundación Saldarriaga Concha, la enfermedad isquémica del corazón es la que más sufre esta población en el país, pues la padecen el $20 \%$ de los hombres y el 18,8 \% de las mujeres mayores de 60 años. También padecen enfermedades crónicas de las vías respiratorias internas (9,5\% en hombres, $12,1 \%$ en mujeres); cerebrovasculares ( $9,4 \%$ en hombres, $7,9 \%$ en mujeres); hipertensivas (4,6\% en hombres, 6,6 \% en mujeres) y Diabetes Mellitus (4,2\% en hombres, 5,3\% en mujeres) (4).

De otra parte, la mortalidad en la población mayor puede atribuirse a enfermedades de larga duración como el cáncer, las afecciones respiratorias crónicas, las cardiopatías, las enfermedades osteomusculares como la artritis y la osteoporosis y los trastornos mentales y neurológicos. De hecho, de estas dos últimas proviene la población mayor del país: la depresión. Otra dificultad a la que se enfrentan los adultos mayores es que en Colombia hay un déficit de personal médico dedicado a atender esta población. Si bien desde 1982 a la fecha se han formado 2.000 gerontólogos en el país, de estos apenas hay $4 \%$ en actividad. Lo anterior significa que actual- 
mente hay 80 geriatras, 30 en formación y la mayoría se encuentran en Bogotá, es decir que, solo hay un especialista por cada 35.000 adultos mayores en Colombia $(4,7)$.

Respecto al abandono, hay aproximadamente 5.750.000 adultos mayores, de ellos unos 995.000 residen en Bogotá y cerca de 400 son abandonadas cada año en la capital. El documento de la Universidad de La Sabana, también revela que la pirámide poblacional del país se ha invertido a tal punto de que hay más gente mayor de 60 años que niños menores de cinco. Se calcula que para el año 2020 "por cada dos adultos mayores habrá un adolescente", según los resultados de la Encuesta de Salud, Bienestar y Envejecimiento, realizada por distintos organismos públicos y privados, entre ellos la Universidad de La Sabana, que consultó a por lo menos 30 mil adultos mayores, habitantes de 250 municipios de Colombia (8).

Debido a las cifras y porcentajes de alto índice de maltrato físico, psicológico y abandono, se expidió una ley de protección al adulto mayor que castiga por abandono y descuido, penaliza a quien ejerce maltrato físico, sicológico y a quienes restrinjan la locomoción de 20 personas mayores de edad. Se trata de la Ley 1850 del 19 de julio de 2017 (9).

Con esta ley se modifica el artículo 229 del Código Penal para establecer que quien someta a condición de abandono y descuido a una persona mayor, con 60 años de edad o más, y afecte sus necesidades de higiene, vestuario, alimentación o salud, incurrirá en penas que pueden ir de 4 a 8 años de prisión y tendrá que pagar multas entre 1 y 5 salarios mínimos mensuales legales vigentes. 
Dispone que el abandono de una persona mayor por parte de una institución a la cual corresponde su cuidado, será causal para cancelación de los permisos de funcionamiento. Además, la institución será sancionada con una multa de 20 salarios mínimos mensuales legales vigentes. Con esta normativa también se establecen penas para quien maltrate física o sicológicamente a las personas mayores de su núcleo familiar. (A estas penas también quedará sometido quien, no siendo miembro del núcleo familiar, sea el encargado del cuidado del adulto mayor y lo maltrate física o sicológicamente) (9).

En cuanto al maltrato de los adultos mayores mediante restricción a la libertad física, la ley penaliza y establece multas contra quien restrinja su libertad de locomoción (esto, sin importar que sea parte de su familia o que esté bajo su tutela).

Esta ley contempla el tema de estrategias, políticas, programas, proyectos y acciones que debe poner en marcha el Estado para los adultos mayores, así como los derechos que tienen las personas mayores en materia de nutrición, habitación, vestuario, afiliación al sistema general de seguridad social en salud, recreación y cultura. De la situación de los adultos mayores en el país, la situación económica y pobreza es quizá una de las razones que más afecta a este grupo poblacional. El país, que si bien comparte la necesidad de reducir la pobreza como propósito de la Cumbre del Milenio, no ha podido avanzar en este objetivo; por el contrario se ha incrementado el número de pobres en el país. Se calcula que la mitad de las personas mayores de 60 años son pobres, al igual que en el resto de América Latina, lo que incrementa su vulnerabilidad, pese a que es una población que mantiene su participación en el mercado laboral, "las personas adultas mayores entre 60 y 79 años continúan parti- 
cipando en el mercado laboral en cerca del 29,9\% de las personas de este grupo de edad".

Por su parte, los ingresos económicos decrecen proporcionalmente con la edad, se estima que estos descienden progresivamente a partir de los 50 años, hasta llegar a un $40 \%$ menos a los 80 años.

Las anteriores cifras evidencian desafíos para la formulación de las políticas públicas culturales, en especial los relacionados a los saberes de acción, los conocimientos y las prácticas culturales, que permiten reconocer a las adultas y adultos mayores como sujetos activos y productivos. Estos retos dan cuenta de la urgente necesidad de considerar el tema como un asunto relevante para el quehacer público y privado, en particular en relación con la capacidad de los individuos, las familias y las organizaciones de identificar y explorar los aportes de esta población en la tradición oral, en la construcción de ciudadanías activas y por tanto en el fortalecimiento de la democracia, la construcción de paz y una verdadera Cultura por el Envejecimiento y la Vejez, con el respaldo de políticas públicas, de sanidad y sociales.

\section{Marco conceptual de la política pública}

Como respuesta a la problemática que presenta esta población, en el 2007 se institucionalizó la política nacional en envejecimiento y vejez 2007-2019. "Es una política pública, de Estado, concertada, que articula a todos los estamentos del gobierno y la sociedad civil en un propósito común: visibilizar e intervenir la situación del envejecimiento y la vejez en el periodo 2007-2019. Ministerio de la Protección Social" (10). 
Su marco conceptual se relaciona con envejecimiento individual y envejecimiento demográfico, los derechos humanos, universales, imprescriptibles, innatos e irrenunciables, envejecimiento activo, protección social integral, riesgo, vulnerabilidad, manejo social del riesgo mediante estrategias de prevención, estrategias de mitigación, y estrategias de superación.

Los elementos aquí descritos son bases fundamentales para que el ejercicio de la política pública tenga un marco conceptual claro en el cual moverse y responder a las necesidades que afecta a este grupo poblacional.

\section{Desafíos de la política}

Los desafíos de la política pública son: "El envejecimiento demográfico, el envejecimiento femenino, la enfermedad, la discapacidad, la dependencia y la calidad de vida de las personas mayores, superar las condiciones de exclusión social, el imaginario cultural adverso y las representaciones sociales discriminatorias del envejecimiento y la vejez, y por último la erradicación del hambre y de la pobreza extrema en la población de persona mayor, en condiciones de vulnerabilidad" (10). Lo anterior, orientado a garantizar un envejecimiento saludable que contribuya a promover condiciones que permitan a las personas tener una vida larga y saludable.

Este desafío es un compromiso de todo el país, los niveles de corresponsabilidad requieren de un monitoreo permanente; de tal manera que el Estado, la familia y la sociedad en general lo interioricen como un valor para poder garantizar una mejor cultura del envejecimiento y calidad de vida para las personas mayores que habitan en Colombia y requieren una pronta atención. 
Tabla 1

Indicadores para el seguimiento de la Política Nacional de Envejecimiento y Vejez (10)

\begin{tabular}{|c|c|c|c|c|}
\hline $\begin{array}{l}\text { Área temática } \\
\text { a evaluar }\end{array}$ & $\begin{array}{l}\text { Nombre del } \\
\text { indicador }\end{array}$ & $\begin{array}{c}\text { Fuente de } \\
\text { información }\end{array}$ & Periodicidad & Responsable \\
\hline \multirow{3}{*}{$\begin{array}{l}\text { Demografía } \\
\text { del } \\
\text { Envejecimiento }\end{array}$} & $\begin{array}{l}\text {-Número de } \\
\text { personas mayores. } \\
\text {-Proporción de } \\
\text { personas mayores. } \\
\text {-Evolución } \\
\text { del número de } \\
\text { personas mayores. }\end{array}$ & $\begin{array}{l}\text { Censos de } \\
\text { población } \\
\text { yproyec- } \\
\text { ciones. }\end{array}$ & Anual & $\begin{array}{l}\text {-DANE } \\
\text {-Entidades } \\
\text { Territoriales } \\
\text {-Ministerios } \\
\text {-Aseguradores }\end{array}$ \\
\hline & $\begin{array}{l}\text { Edad media de la } \\
\text { población. }\end{array}$ & $\begin{array}{l}\text { Censos de } \\
\text { población } \\
\text { yproyec- } \\
\text { ciones. }\end{array}$ & Anual & $\begin{array}{l}\text {-DANE } \\
\text {-Entidades } \\
\text { Territoriales } \\
\text {-Ministerios } \\
\text {-Aseguradores }\end{array}$ \\
\hline & $\begin{array}{l}\text { Ritmo de creci- } \\
\text { miento de la } \\
\text { población adulta }\end{array}$ & $\begin{array}{l}\text { Censos de } \\
\text { población } \\
\text { yproyec- } \\
\text { ciones. }\end{array}$ & Anual & $\begin{array}{l}\text {-DANE } \\
\text {-Entidades } \\
\text { Territoriales } \\
\text {-Ministerios } \\
\text {-Aseguradores }\end{array}$ \\
\hline
\end{tabular}

Fuente: tomada de la política de envejecimiento y vejez (2007-2019)

Los indicadores permiten hacer seguimiento y control a cada una de las áreas temáticas a fin de mirar el cumplimiento de los objetivos planteados en el marco de la política. La Tabla 1 es un ejemplo donde se puede apreciar los elementos a tener en cuenta en el seguimiento.

\section{Principios de la política}

Los principios que resalta la política están enfocados hacia la universalidad, equidad, dignidad, solidaridad intergeneracional, justicia social distributiva, participación social y enfoque diferencial.

Los principios enmarcados en la política son pertinentes porque con su enfoque filosófico responden al favorecimiento de las personas mayores con el ánimo de incluirlas y hacerlas partícipes 
como sujetos activos que son, en la materialización de proyectos y programas que redunden en el bienestar colectivo (9).

\section{Objetivos generales de la política}

La política contempla dos grandes objetivos:

1. "Incidir de manera activa, a nivel intersectorial y territorial sobre las condiciones de desarrollo social, económico y cultural de los individuos, la familia y la sociedad, como medio para propiciar que los viejos de hoy y del futuro alcancen una vejez digna, saludable e integrada, dentro del marco de la promoción, prevención y restitución de los derechos humanos".

2. "Crear condiciones para el envejecimiento de la población, entendido como el derecho de toda la población a una vida digna, larga y saludable, reconociendo la trascendencia de la corresponsabilidad individual, familiar y social en este proceso".

Las dos metas que plantea la política pública son un pilar que le da sostenimiento puesto que contempla el enfoque social de derechos mediante el desarrollo de las esferas económicas, sociales y culturales (10).

\section{Ejes de la política}

Eje1: Promoción y garantía de los derechos humanos de las personas mayores

Eje 2: Protección Social Integral

Eje 3: Envejecimiento Activo 
Eje 4: Formación del Talento Humano e Investigación

\section{Decretos y leyes posteriores a la Política}

Decreto 3039 de 2007 Política nacional de salud pública "enfrentar los retos del envejecimiento poblacional y la transición demográfica".

Ley 1251 de 2008 protección, promoción y defensa derechos de los mayores (12).

Ley 1171 de 2007 beneficios (62 años) (11).

Ley 1315 de 2009 condiciones mínimas que dignifiquen la estadía de los adultos mayores en los centros de día e instituciones de atención (13).

\section{Organismos de Vigilancia y Control}

Les corresponde en ejercicio de sus funciones, vigilar y controlar la gestión integral de la Política de Envejecimiento y Vejez, monitorear el logro de los resultados y aplicar las sanciones correspondientes por incumplimiento (10).

\section{Reflexiones finales}

La situación de los mayores en el país, en aspectos económicos y de pobreza, es quizá una de las razones que más afecta a este grupo poblacional en el país. Se quiere reducir la pobreza como propósito de la Cumbre del Milenio, sin embargo no se ha podido avanzar en este objetivo, por el contrario se ha incrementado el número de pobres en Colombia. 
Hasta este momento no hay una conciencia clara del envejecimiento, solo el rechazo permanente por aumento de edad.

Sin embargo, con base en la encuesta de Demografía y salud (ENDS), 2010 incluye por primera vez el tema de vejez con la denominación Adulto mayor, considerando que el envejecimiento y la mayor longevidad poblacional constituyen una realidad en el mundo, específicamente en América latina y puntualmente en nuestro país: Colombia (6).

La esperanza de vida es hoy de 76 años, siendo esta superior en las mujeres que en los hombres. En su mayoría las personas mayores viven en zonas urbanas y más del 60 \% se concentran en Bogotá, por ser la capital, así como en los departamentos de Antioquia, Valle, Cundinamarca, Santander, Atlántico, Bolívar y Tolima.

Como no existe en la mayoría de las personas mayores conciencia de sus derechos, es necesario realizar procesos formativos que fomenten su empoderamiento como ciudadanos, su autonomía y la participación activa en organizaciones.

Con la implementación de la política pública de envejecimiento y vejez muchas necesidades de esta población han disminuido, contribuyendo a la mejora de la calidad de vida de las personas mayores.

Las políticas y la legislación no pueden quedarse en el papel, deben trascender e impactar positivamente a la población envejeciente y a la que ya es mayor de 60 y más años.

Por lo anterior se requiere de una cultura del envejecimiento; educación, medios de comunicación, recursos humanos cuali- 
ficados, investigaciones y sistematización, divulgación de experiencias exitosas y vigilancia permanente de la política, ya que el aumento de la población encierra grandes retos, los que deben ser asumidos en forma creativa y positiva, favoreciendo un mayor desarrollo y enriquecimiento humano, tanto para quienes son mayores como para las personas que conviven con ellos. Así, el fenómeno del envejecimiento de la población debe ser entendido no solo como un cambio estadístico demográfico, sino como una profunda transformación de la sociedad, que involucra aspectos variados y complejos que obligan a replantear estrategias desde las políticas públicas, de sanidad, relaciones familiares, necesidades culturales y materiales, desafiando las capacidades estructurales y funcionales de todos los agentes sociales involucrados.

\section{REFERENCIAS BIBLIOGRÁFICAS}

1. Durán DM, Valderrama LJ, Uribe AF, Uribe JM. Integración social y habilidades funcionales en adultos mayores. Univ. Psychol. 2008;7(1):263-270. Disponible en: http://sparta. javeriana.edu.co/psicologia/publicaciones/actualizarrevista/ archivos/Vo7No118.PDF

2. Organización Mundial de la Salud. Hombres, envejecimiento y salud. 2001. Disponible en: http://www.redadultosmayores. com. ar/buscador/files/SALUDo08.pdf

3. Comisión Económica para América Latina y el Caribe (CEPAL), 2015. Proyecciones de población. Chile. Disponible en: https:// www.cepal.org/es.

4. Flórez C, Villar L, Puerta N, Berrocal L. El proceso de envejecimiento de la población colombiana: 1985-2015, Misión Colombia. 2015. Editorial Fundación Saldarriaga Concha. Bogotá, D.C. Colombia 67 p.

5. Minsalud - Colciencias. Encuesta Sabe Colombia. Estudio 
Nacional de Salud Bienestar y Envejecimiento, 2015. Disponible en: https://www.minsalud.gov.co/sites/rid/Lists/BibliotecaDigital/RIDE/VS/ED/GCFI/Resumen-Ejecutivo-Encuesta-SABE.pdf

6. Profamilia. Ministerio encuesta Nacional de Demografía y Salud. ENDS, 2013. Disponible en: https://profamilia.org.co/ investigaciones/ends/

7. Ortegón F. Informe de Envejecimiento. ACGG y Universidad de la Sabana. 2017. Disponible en: http://acgg.org.co/

8. Ley 1850 del 19 de julio de 2017.

9. Ministerio de la Protección Social. República de Colombia. Política Nacional de Envejecimiento. 2007-2019. Citado el 7 de octubre de 2016. Disponible en: https://www.minsalud.gov.co/ Documentos\%20y\%20Publicaciones/POL\%C3\%8DTICA\%20 NACIONAL\%20DE\%20ENVEJECIMIENTO\%20Y\%20VEJEZ.pdf

10. Congreso de Colombia, Ley1251 de 2008, Protección, Promoción y Defensa de los Derechos de los Adultos Mayores. Disponible en: http://www.alcaldiabogota.gov.co/sisjur/normas/Norma1. jsp?i=33964\#0

11. Congreso de Colombia, Ley 1171 de 2007, Beneficios (62 años). Disponible en: http://www.alcaldiabogota.gov.co/sisjur/ normas/Norma1.jsp?i=27907

12. Congreso de Colombia, Ley 1315 de 2009. Condiciones mínimas que dignifiquen la estadía de los adultos mayores en los centros de día e instituciones de atención. Disponible en: http://www. alcaldiabogota.gov.co/sisjur/normas/Norma1.jsp?i=33964\#0

\section{Cómo citar este artículo}

Cardeño Sanmiguel GM, Morales Castro YR. Demografía del envejecimiento y vejez en el marco de la política pública 2007-2019. In Quintero Cruz MV, Pinillos Patiño Y, Herazo Beltrán AY, Vidarte Claros JA, Cardeño Sanmiguel GM, Morales Castro YR. Ejercicio físico para la condición física funcional en el adulto mayor: Estrategia de intervención. Barranquilla: Universidad Simón Bolívar; 2017. p. 13-29. 